\title{
CERTAIN SEQUENCE OF FUNCTIONS INVOLVING GENERALIZED HYPERGEOMETRIC FUNCTIONS
}

\author{
P. AGARWAL, S. JAIN, İ. O. KIYMAZ, M. CHAND, S.K.Q. AL-OMARI \\ (Communicated by Ali ÇEVİK)
}

\begin{abstract}
A remarkably large number of operational techniques have drawn the attention of several researchers in the study of sequence of functions and polynomials. In this sequel, here, we aim to introduce a new sequence of functions involving the generalized Gauss hypergeometric function by using operational techniques. Some generating relations and finite summation formula of the sequence presented here are also considered.
\end{abstract}

\section{INTRODUCTION}

In recent years, operational techniques have been attract the attention of many researchers due to their importance and applications in various sub-fields of analysis (see [5], [9],[8], [17],Srivastava and Singh[20], Mittal[11, 12, 13], Chandal[6, 7], Srivastava[16], Joshi and Parjapat[10],Patil and Thakare[15] and Srivastava and Singh[19]). In the sequels a remarkably large number of sequence of functions involving a variety of special functions have been developed by many authors (see, for example, [19]; for a very recent work, see also [18]). In the present study we aim to develop a new sequence of functions involving the $F_{p}^{(\alpha, \beta)}($.$) by using operational$ techniques, which are expressed in terms of the generalized Gauss hypergeometric function. Furthermore, some generating relations and finite summation formula are also obtained.

For our purpose, we begin by recalling some known functions and earlier works.

In 1971, Mittal [11] gives the Rodrigues formula for the generalized Lagurre polynomials defined as:

$$
T_{k n}^{(\alpha)}(x)=\frac{1}{n !} x^{-\alpha} \exp \left(p_{k}(x)\right) D^{n}\left[x^{\alpha+n} \exp \left(-p_{k}(x)\right)\right]
$$

where $p_{k}(x)$ is a polynomial in $x$ of degree $k$.

Mittal [12] also proved the following relation for (1.1) defined as:

$$
T_{k n}^{(\alpha+s-1)}(x)=\frac{1}{n !} x^{-\alpha-n} \exp \left(p_{k}(x)\right) T_{s}^{n}\left[x^{\alpha} \exp \left(-p_{k}(x)\right)\right]
$$

Date: Received: August 13, 2015; Accepted: October 9, 2015.

2010 Mathematics Subject Classification. 33E10, 33E12, 33E99, 44A45.

Key words and phrases. Special function, generating relations, generalized Gauss hypergeomtric functions, Sequence of function,finite summation formula. 
where $s$ is constant and $T_{s} \equiv x(s+x D)$.

In this sequel, in 1979, Srivastava and Singh [19] studied a sequence of functions $V_{n}^{(\alpha)}(x ; a, k, s)$ defined as:

$$
V_{n}^{(\alpha)}(x ; a, k, s)=\frac{x^{-\alpha}}{n !} \exp \left\{p_{k}(x)\right\} \theta^{n}\left[x^{\alpha} \exp \left\{-p_{k}(x)\right\}\right]
$$

By employing the operator $\theta \equiv x^{a}(s+x D)$, where $s$ is constant and $p_{k}(x)$ is a polynomial in $x$ of degree $k$.

A new sequence of function $\left\{V_{n}^{(\lambda, \mu ; \nu ; \rho, \sigma ; p ; \alpha)}(x ; a, k, s)\right\}_{n=0}^{\infty}$ is introduced in this paper as:

$$
\begin{aligned}
V_{n}^{(\lambda, \mu ; \nu ; \rho, \sigma ; p ; \alpha)}(x ; a, k, s) & =\frac{1}{n !} x^{-\alpha} F_{p}^{(\rho, \sigma)}\left[\lambda, \mu ; \nu ; p_{k}(x)\right] \\
& \times\left(T_{x}^{a, s}\right)^{n}\left\{x^{\alpha} F_{p}^{(\rho, \sigma)}\left[\lambda, \mu ; \nu ;-p_{k}(x)\right]\right\},
\end{aligned}
$$

where $T_{x}^{a, s} \equiv x^{a}(s+x D), D \equiv \frac{d}{d x}, a$ and $s$ are constants, $k$ is finite and nonnegative integer, $p_{k}(x)$ is a polynomial in $x$ of degree $k$ and $F_{p}^{(\rho, \sigma)}[\lambda, \mu ; \nu ; x]$ is a generalized Gauss hypergeometric functions of one variables. For the sake of completeness, we define this function here (for more detail see [14]):

$$
F_{p}^{(\rho, \sigma)}(\lambda, \mu ; \nu ; x)=\sum_{n=0}^{\infty}(\lambda)_{n} \frac{B_{p}^{(\rho, \sigma)}(\mu+n, \nu-\mu)}{B(\mu, \nu-\mu)} \frac{x^{n}}{n !} ; \quad(|x|<1),
$$

where $\min (\Re(\rho), \Re(\sigma))>0 ; \Re(\nu)>\Re(\mu)>0$ and $\Re(p) \geq 0$ and $B_{p}^{(\rho, \sigma)}(x, y)$ is generalized Beta type function, which is introduced and studied by Özergin et al. [23] in their paper and defined by (see, e.g., [23, p. 4602, Eq.(4)]; see also, [22, p.32, Chapter 4.]):

$$
B_{p}^{(\rho, \sigma)}(x, y):=\int_{0}^{1} t^{x-1}(1-t)^{y-1}{ }_{1} F_{1}\left(\rho ; \sigma ; \frac{-p}{t(1-t)}\right) d t
$$

$$
\left(\Re(p) \geq 0 ; \min (\Re(x), \Re(y), \Re(\rho), \Re(\sigma))>0 \text { and } B_{0}^{(\rho, \sigma)}(x, y)=B(x, y)\right),
$$

where $B(x, y)$ is a well known Euler's Beta function defined by:

$$
B(x, y):=\int_{0}^{1} t^{x-1}(1-t)^{y-1} d t(\Re(x)>0, \Re(y)>0) .
$$

Some generating relations and finite summation formula of class of polynomials or sequence of function have been obtained by using the properties of the differential operators. $T_{x}^{a, s} \equiv x^{a}(s+x D)$, where $D \equiv \frac{d}{d x}$, is based on the work of Mittal[13], Patil and Thakare[15], Srivastava and Singh [19].

Some useful operational techniques are given below:

$$
\begin{gathered}
\exp \left(t T_{x}^{a, s}\right)\left(x^{\beta} f(x)\right)=x^{\beta}\left(1-a x^{a} t\right)^{-\left(\frac{\beta+s}{a}\right)} f\left(x\left(1-a x^{a} t\right)^{-1 / a}\right), \\
\exp \left(t T_{x}^{a, s}\right)\left(x^{\alpha-a n} f(x)\right)=x^{\alpha}(1+a t)^{-1+\left(\frac{\alpha+s}{a}\right)} f\left(x(1+a t)^{1 / a}\right), \\
\left(T_{x}^{a, s}\right)^{n}(x u v)=x \sum_{m=0}^{\infty}\left(\begin{array}{c}
n \\
m
\end{array}\right)\left(T_{x}^{a, s}\right)^{n-m}(v)\left(T_{x}^{a, 1}\right)^{m}(u),
\end{gathered}
$$




$$
\begin{gathered}
(1+x D)(1+a+x D) \ldots(1+(m-1) a+x D) x^{\beta-1}=a^{m}\left(\frac{\beta}{a}\right)_{m} x^{\beta-1}, \\
(1-a t)^{\frac{-\alpha}{a}}=(1-a t)^{\frac{-\beta}{a}} \sum_{m=0}^{\infty}\left(\frac{\alpha-\beta}{a}\right)_{m} \frac{(a t)^{m}}{m !} .
\end{gathered}
$$

\section{Generating Relations}

In this section, we present some generating relations involving the $F_{p}^{(\alpha, \beta)}($.$) .$ First generating relation:

$$
\begin{aligned}
& \sum_{n=0}^{\infty} V_{n}^{(\lambda, \mu ; \nu ; \rho, \sigma ; p ; \alpha)}(x ; a, k, s) x^{-a n} t^{n}=(1-a t)^{-\left(\frac{\alpha+s}{a}\right)} \\
& \quad \times F_{p}^{(\rho, \sigma)}\left[\lambda, \mu ; \nu ; p_{k}(x)\right] F_{p}^{(\rho, \sigma)}\left[\lambda, \mu ; \nu ;-p_{k}\left(x(1-a t)^{-1 / a}\right)\right] .
\end{aligned}
$$

\section{Second generating relation:}

$$
\begin{aligned}
& \sum_{n=0}^{\infty} V_{n}^{(\lambda, \mu ; \nu ; \rho, \sigma ; p ; \alpha-a n)}(x ; a, k, s) x^{-a n} t^{n}=(1+a t)^{-1+\left(\frac{\alpha+s}{a}\right)} \\
& \quad \times F_{p}^{(\rho, \sigma)}\left[\lambda, \mu ; \nu ; p_{k}(x)\right] F_{p}^{(\rho, \sigma)}\left[\lambda, \mu ; \nu ;-p_{k}\left(x(1+a t)^{1 / a}\right)\right] .
\end{aligned}
$$

\section{Third generating relation:}

$$
\begin{aligned}
& \sum_{m=0}^{\infty}\left(\begin{array}{c}
m+n \\
n
\end{array}\right) V_{m+n}^{(\lambda, \mu ; \nu ; \rho, \sigma ; p ; \alpha)}(x ; a, k, s) x^{-a m} t^{m}=(1-a t)^{-\left(\frac{\alpha+s}{a}\right)} \\
& \quad \times \frac{F_{p}^{(\rho, \sigma)}\left[\lambda, \mu ; \nu ; p_{k}(x)\right]}{F_{p}^{(\rho, \sigma)}\left[\lambda, \mu ; \nu ; p_{k}\left(x(1-a t)^{-1 / a}\right)\right]} \\
& \quad \times V_{n}^{(\lambda, \mu ; \nu ; \rho, \sigma ; p ; \alpha)}\left(x(1-a t)^{-1 / a} ; a, k, s\right) .
\end{aligned}
$$

Proof of first generating relation

From (1.4), we obtain

$$
\begin{aligned}
\sum_{n=0}^{\infty} V_{n}^{(\lambda, \mu ; \nu ; \rho, \sigma ; p ; \alpha)}(x ; a, k, s) t^{n}=x^{-\alpha} F_{p}^{(\rho, \sigma)}\left[\lambda, \mu ; \nu ; p_{k}(x)\right] \\
\times \exp \left(t T_{x}^{a, s}\right)\left\{x^{\alpha} F_{p}^{(\rho, \sigma)}\left[\lambda, \mu ; \nu ;-p_{k}(x)\right]\right\} .
\end{aligned}
$$

Using operational technique (1.6), above equation (2.4) is reduces to

$$
\begin{aligned}
& \sum_{n=0}^{\infty} V_{n}^{(\lambda, \mu ; \nu ; \rho, \sigma ; p ; \alpha)}(x ; a, k, s) t^{n}=\left(1-a x^{a} t\right)^{-\left(\frac{\alpha+s}{a}\right)} \\
& \quad \times F_{p}^{(\rho, \sigma)}\left[\lambda, \mu ; \nu ; p_{k}(x)\right] F_{p}^{(\rho, \sigma)}\left[\lambda, \mu ; \nu ;-p_{k}\left(x\left(1-a x^{a} t\right)^{-1 / a}\right)\right] .
\end{aligned}
$$

Replacing $t$ by $t x^{-a},(2.1)$ is obtained. 


\section{Proof of second generating relation}

Again from (1.4), we have

$$
\begin{gathered}
\sum_{n=0}^{\infty} x^{-a n} V_{n}^{(\lambda, \mu ; \nu ; \rho, \sigma ; p ; \alpha-a n)}(x ; a, k, s) t^{n}=x^{-\alpha} F_{p}^{(\rho, \sigma)}\left[\lambda, \mu ; \nu ; p_{k}(x)\right] \\
\times \exp \left(t T_{x}^{a, s}\right)\left\{x^{\alpha-a n} F_{p}^{(\rho, \sigma)}\left[\lambda, \mu ; \nu ;-p_{k}(x)\right]\right\} .
\end{gathered}
$$

Applying the operational technique (1.7), we get

$$
\begin{aligned}
& \sum_{n=0}^{\infty} x^{-a n} V_{n}^{(\lambda, \mu ; \nu ; \rho, \sigma ; p ; \alpha-a n)}(x ; a, k, s) t^{n}=(1+a t)^{\frac{\alpha+s}{a}-1} \\
& \times F_{p}^{(\rho, \sigma)}\left[\lambda, \mu ; \nu ; p_{k}(x)\right] F_{p}^{(\rho, \sigma)}\left[\lambda, \mu ; \nu ;-p_{k}\left(x(1+a t)^{1 / a}\right)\right],
\end{aligned}
$$

which is desired.

\section{Proof of third generating relation}

We can write (1.4) as

$$
\left(T_{x}^{a, s}\right)^{n}\left[x^{\alpha} F_{p}^{(\rho, \sigma)}\left[\lambda, \mu ; \nu ;-p_{k}(x)\right]\right]=n ! x^{\alpha} \frac{V_{n}^{(\lambda, \mu ; \nu ; \rho, \sigma ; p ; \alpha)}(x ; a, k, s)}{F_{p}^{(\rho, \sigma)}\left[\lambda, \mu ; \nu ; p_{k}(x)\right]} .
$$

Thus we obtain

$$
\begin{gathered}
\exp \left(t\left(T_{x}^{a, s}\right)\right)\left\{\left(T_{x}^{a, s}\right)^{n}\left[x^{\alpha} F_{p}^{(\rho, \sigma)}\left[\lambda, \mu ; \nu ;-p_{k}(x)\right]\right]=n ! \exp \left(t T_{x}^{a, s}\right)\right. \\
\times\left[x^{\alpha} \frac{V_{n}^{(\lambda, \mu ; \nu ; \rho, \sigma ; p ; \alpha)}(x ; a, k, s)}{F_{p}^{(\rho, \sigma)}\left[\lambda, \mu ; \nu ; p_{k}(x)\right]}\right] \\
\sum_{m=0}^{\infty} \frac{t^{m}}{m !}\left(T_{x}^{a, s}\right)^{m+n}\left\{x^{\alpha} F_{p}^{(\rho, \sigma)}\left[\lambda, \mu ; \nu ;-p_{k}(x)\right]\right\}=n ! \exp \left(t T_{x}^{a, s}\right) \\
\times\left\{x^{\alpha} \frac{V_{n}^{(\lambda, \mu ; \nu ; \rho, \sigma ; p ; \alpha)}(x ; a, k, s)}{F_{p}^{(\rho, \sigma)}\left[\lambda, \mu ; \nu ; p_{k}(x)\right]}\right\} .
\end{gathered}
$$

Using the operational technique (1.6), above equation can be written as

$$
\begin{aligned}
& \sum_{m=0}^{\infty} \frac{t^{m}}{m !}\left(T_{x}^{a, s}\right)^{m+n}\left[x^{\alpha} F_{p}^{(\rho, \sigma)}\left[\lambda, \mu ; \nu ;-p_{k}(x)\right]\right]=n ! x^{\alpha} \\
& \quad \times\left(1-a x^{a} t\right)^{-\left(\frac{\alpha+s}{a}\right)} \frac{V_{n}^{(\lambda, \mu ; \nu ; \rho, \sigma ; p ; \alpha)}\left(x\left(1-a x^{a} t\right)^{-1 / a} ; a, k, s\right)}{F_{p}^{(\rho, \sigma)}\left[\lambda, \mu ; \nu ; p_{k}\left(x\left(1-a x^{a} t\right)^{-1 / a}\right)\right]} .
\end{aligned}
$$

Using (2.9), above equation gives

$$
\begin{aligned}
& \sum_{m=0}^{\infty} \frac{t^{m}(m+n) !}{m ! n !} x^{\alpha} \frac{V_{m+n}^{(\lambda, \mu ; \nu ; \rho, \sigma ; p ; \alpha)}(x ; a, k, s)}{F_{p}^{(\rho, \sigma)}\left[\lambda, \mu ; \nu ;-p_{k}(x)\right]}=x^{\alpha} \\
& \quad \times\left(1-a x^{a} t\right)^{-\left(\frac{\alpha+s}{a}\right)} \frac{V_{n}^{(\lambda, \mu ; \nu ; \rho, \sigma ; p ; \alpha)}\left(x\left(1-a x^{a} t\right)^{-1 / a} ; a, k, s\right)}{F_{p}^{(\rho, \sigma)}\left[\lambda, \mu ; \nu ;-p_{k}\left(x\left(1-a x^{a} t\right)^{-1 / a}\right)\right]} .
\end{aligned}
$$


Therefore

$$
\begin{aligned}
& \sum_{m=0}^{\infty}\left(\begin{array}{c}
m+n \\
n
\end{array}\right) V_{m+n}^{(\lambda, \mu ; \nu ; \rho, \sigma ; p ; \alpha)}(x ; a, k, s) t^{m}=\left(1-a x^{a} t\right)^{-\left(\frac{\alpha+s}{a}\right)} \\
& \quad \times \frac{F_{p}^{(\rho, \sigma)}\left[\lambda, \mu ; \nu ; p_{k}(x)\right] V_{n}^{(\lambda, \mu ; \nu ; \rho, \sigma ; p ; \alpha)}\left(x\left(1-a x^{a} t\right)^{-1 / a} ; a, k, s\right)}{F_{p}^{(\rho, \sigma)}\left[\lambda, \mu ; \nu ; p_{k}\left(x\left(1-a x^{a} t\right)^{-1 / a}\right)\right]} .
\end{aligned}
$$

Replacing $t$ by $t x^{-a}$, this gives the result (2.3).

Remark 2.1. If we give some suitable parametric replacement in (2.1), (2.2) and (2.3) respectively, then we can see the known results (see $[5,6,7,8,9,10,11,12$, $15,18,17,19,20])$.

\section{Finite Summation Formulas}

Here, we study some finite summation formulas involving the $F_{p}^{(\alpha, \beta)}($.

First finite summation formula:

$$
V_{n}^{(\lambda, \mu ; \nu ; \rho, \sigma ; p ; \alpha)}(x ; a, k, s)=\sum_{m=0}^{n} \frac{1}{m !}\left(a x^{a}\right)^{m}\left(\frac{\alpha}{a}\right)_{m} V_{n-m}^{(\lambda, \mu ; \nu ; \rho, \sigma ; p ; 0)}(x ; a, k, s) .
$$

\section{Second finite summation formula:}

$$
\begin{aligned}
V_{n}^{(\lambda, \mu ; \nu ; \rho, \sigma ; p ; \alpha)}(x ; a, k, s) & =\sum_{m=0}^{n} \frac{1}{m !}\left(a x^{a}\right)^{m}\left(\frac{\alpha-\beta}{a}\right)_{m} \\
& \times V_{n-m}^{(\lambda, \mu ; \nu ; \rho, \sigma ; p ; \beta)}(x ; a, k, s) .
\end{aligned}
$$

\section{Proof of first finite summation formula}

From equation (1.4), we have

$$
\begin{aligned}
V_{n}^{(\lambda, \mu ; \nu ; \rho, \sigma ; p ; \alpha)}(x ; a, k, s) & =\frac{1}{n !} x^{-\alpha} F_{p}^{(\rho, \sigma)}\left[\lambda, \mu ; \nu ; p_{k}(x)\right] \\
& \times\left(T_{x}^{a, s}\right)^{n}\left\{x x^{\alpha-1} F_{p}^{(\rho, \sigma)}\left[\lambda, \mu ; \nu ;-p_{k}(x)\right]\right\} .
\end{aligned}
$$

Using the operational technique (1.8), we have

$$
\begin{aligned}
V_{n}^{(\lambda, \mu ; \nu ; \rho, \sigma ; p ; \alpha)} & (x ; a, k, s)=\frac{1}{n !} x^{-\alpha} F_{p}^{(\rho, \sigma)}\left[\lambda, \mu ; \nu ; p_{k}(x)\right] x \\
& \times \sum_{m=0}^{\infty}\left(\begin{array}{c}
n \\
m
\end{array}\right)\left(T_{x}^{a, s}\right)^{n-m}\left\{F_{p}^{(\rho, \sigma)}\left[\lambda, \mu ; \nu ;-p_{k}(x)\right]\right\}\left(T_{x}^{a, 1}\right)^{m}\left(x^{\alpha-1}\right) \\
& =\frac{1}{n !} x^{-\alpha} F_{p}^{(\rho, \sigma)}\left[\lambda, \mu ; \nu ; p_{k}(x)\right] x \sum_{m=0}^{\infty} \frac{n !}{m !(n-m) !} x^{a(n-m)} \\
& \times[(s+x D)(s+a+x D)(s+2 a+x D) \ldots(s+(n-m-1) a+x D)] \\
& \times F_{p}^{(\rho, \sigma)}\left[\lambda, \mu ; \nu ;-p_{k}(x)\right] x^{a m} \\
& \times[(1+x D)(1+a+x D)(1+2 a+x D) \ldots(1+(m-1) a+x D)]\left(x^{\alpha-1}\right) .
\end{aligned}
$$


Using the result (1.9), we have

$$
\begin{aligned}
V_{n}^{(\lambda, \mu ; \nu ; \rho, \sigma ; p ; \alpha)}(x ; a, k, s)=F_{p}^{(\rho, \sigma)}\left[\lambda, \mu ; \nu ; p_{k}(x)\right] \sum_{m=0}^{n} \frac{1}{m !(n-m) !} x^{a n} \\
\times \prod_{i=0}^{n-m-1}(s+i a+x D)\left\{F_{p}^{(\rho, \sigma)}\left[\lambda, \mu ; \nu ;-p_{k}(x)\right]\right\} a^{m}\left(\frac{\alpha}{a}\right)_{m} .
\end{aligned}
$$

Putting $\alpha=0$ and replacing $n$ by $n-m$ in (3.3), we get

$$
\begin{aligned}
V_{n-m}^{(\lambda, \mu ; \nu ; \rho, \sigma ; p ; 0)}(x ; a, k, s)=\frac{1}{(n-m) !} F_{p}^{(\rho, \sigma)}\left[\lambda, \mu ; \nu ; p_{k}(x)\right] \\
\times\left(T_{x}^{a, s}\right)^{n-m}\left\{F_{p}^{(\rho, \sigma)}\left[\lambda, \mu ; \nu ;-p_{k}(x)\right]\right\} \\
\Rightarrow \frac{1}{(n-m) !}\left(T_{x}^{a, s}\right)^{n-m}\left\{F_{p}^{(\rho, \sigma)}\left[\lambda, \mu ; \nu ;-p_{k}(x)\right]\right\} \\
=\frac{V_{n-m}^{(\lambda, \mu ; \nu ; \rho, \sigma ; p ; 0)}(x ; a, k, s)}{F_{p}^{(\rho, \sigma)}\left[\lambda, \mu ; \nu ; p_{k}(x)\right]} .
\end{aligned}
$$

This gives

$$
\begin{gathered}
\frac{1}{(n-m) !} \prod_{i=0}^{n-m-1}(s+i a+x D)\left\{F_{p}^{(\rho, \sigma)}\left[\lambda, \mu ; \nu ;-p_{k}(x)\right]\right\} \\
=x^{a(m-n)} \frac{V_{n-m}^{(\lambda, \mu ; \nu ; \rho, \sigma ; p ; 0)}(x ; a, k, s)}{F_{p}^{(\rho, \sigma)}\left[\lambda, \mu ; \nu ; p_{k}(x)\right]} .
\end{gathered}
$$

From equations (3.5) and (3.8) we have the main result.

\section{Proof of second finite summation formula}

Equation (1.4) can be written as

$$
\begin{aligned}
\sum_{n=0}^{\infty} V_{n}^{(\lambda, \mu ; \nu ; \rho, \sigma ; p ; \alpha)} & (x ; a, k, s) t^{n}=x^{-\alpha} F_{p}^{(\rho, \sigma)}\left[\lambda, \mu ; \nu ; p_{k}(x)\right] \\
& \times \exp \left(t T_{x}^{(a, s)}\right)\left\{x^{\alpha} F_{p}^{(\rho, \sigma)}\left[\lambda, \mu ; \nu ;-p_{k}(x)\right]\right\}
\end{aligned}
$$

Applying the equation (1.6) in the equation (3.9), we have

$$
\begin{aligned}
\sum_{n=0}^{\infty} V_{n}^{(\lambda, \mu ; \nu ; \rho, \sigma ; p ; \alpha)} & (x ; a, k, s) t^{n}=\left(1-a x^{a} t\right)^{-\left(\frac{\alpha+s}{a}\right)} F_{p}^{(\rho, \sigma)}\left[\lambda, \mu ; \nu ; p_{k}(x)\right] \\
& \times F_{p}^{(\rho, \sigma)}\left[\lambda, \mu ; \nu ;-p_{k}\left(x\left(1-a x^{a} t\right)^{-1 / a}\right)\right]
\end{aligned}
$$


Applying the result from the equation (1.12) the equation (3.10) is reduced to

$$
\begin{aligned}
\sum_{n=0}^{\infty} & V_{n}^{(\lambda, \mu ; \nu ; \rho, \sigma ; p ; \alpha)}(x ; a, k, s) t^{n}=\left(1-a x^{a} t\right)^{-\left(\frac{\beta+s}{a}\right)} \\
& \times \sum_{m=0}^{\infty}\left(\frac{\alpha-\beta}{a}\right)_{m} \frac{\left(a x^{a} t\right)^{m}}{m !} F_{p}^{(\rho, \sigma)}\left[\lambda, \mu ; \nu ; p_{k}(x)\right] \\
& \times F_{p}^{(\rho, \sigma)}\left[\lambda, \mu ; \nu ;-p_{k}\left(x\left(1-a x^{a} t\right)^{-1 / a}\right)\right] \\
= & \sum_{m=0}^{\infty}\left(\frac{\alpha-\beta}{a}\right)_{m} \frac{\left(a x^{a} t\right)^{m}}{m !} x^{-\beta} F_{p}^{(\rho, \sigma)}\left[\lambda, \mu ; \nu ; p_{k}(x)\right] \exp \left(t T_{x}^{a, s}\right) \\
& \times\left\{x^{\beta} F_{p}^{(\rho, \sigma)}\left[\lambda, \mu ; \nu ;-p_{k}(x)\right]\right\} \\
= & \sum_{m=0}^{\infty} \sum_{n=0}^{\infty}\left(\frac{\alpha-\beta}{a}\right)_{m} \frac{\left(a x^{a}\right)^{m} t^{n+m}}{m ! n !} x^{-\beta} F_{p}^{(\rho, \sigma)}\left[\lambda, \mu ; \nu ; p_{k}(x)\right]\left(T_{x}^{a, s}\right)^{n} \\
& \times\left\{x^{\beta} F_{p}^{(\rho, \sigma)}\left[\lambda, \mu ; \nu ;-p_{k}(x)\right]\right\} \\
= & \sum_{n=0}^{\infty} \sum_{m=0}^{n}\left(\frac{\alpha-\beta}{a}\right)_{m} \frac{\left(a x^{a}\right)^{m} t^{n}}{m !(n-m) !} x^{-\beta} F_{p}^{(\rho, \sigma)}\left[\lambda, \mu ; \nu ; p_{k}(x)\right]\left(T_{x}^{a, s}\right)^{n-m} \\
& \times\left\{x^{\beta} F_{p}^{(\rho, \sigma)}\left[\lambda, \mu ; \nu ;-p_{k}(x)\right]\right\} .
\end{aligned}
$$

Now equating the coefficient of $t^{n}$, we get

$$
\begin{aligned}
& V_{n}^{(\lambda, \mu ; \nu ; \rho, \sigma ; p ; \alpha)}(x ; a, k, s)=\sum_{m=0}^{n}\left(\frac{\alpha-\beta}{a}\right)_{m} \frac{\left(a x^{a}\right)^{m}}{m !(n-m) !} x^{-\beta} \\
& \quad \times F_{p}^{(\rho, \sigma)}\left[\lambda, \mu ; \nu ; p_{k}(x)\right]\left(T_{x}^{a, s}\right)^{n-m}\left\{x^{\beta} F_{p}^{(\rho, \sigma)}\left[\lambda, \mu ; \nu ;-p_{k}(x)\right]\right\} .
\end{aligned}
$$

Using the equation (1.4) in (3.12), we have the result (3.2).

\section{Special Cases and Conclusion}

(I) All the results established in equation (2.1), (2.2), (2.3), (3.1) and (3.2) can be reduced in to the known works (see, $[5,2,3,4]$ ) by assigning suitable value to the parameters in generalized Gauss hypergeometric function $F_{p}^{(\alpha, \beta)}($.$) .$

(II) If we apply the Wright function $W(\alpha, \delta ; z)$ very special case of hypergeomtric function ${ }_{p} F_{q}$; all the results established in equation (2.1), (2.2), (2.3), (3.1) and (3.2) reduced to the work of Joshi and Prajapati [On New Sequence of Functions and Their MATLAB Computation].

Now, we conclude present investigate by remarking that by using our main sequence formula we presented some generating relations and finite summation formula of the sequence here. All results of this paper are important due to presence of $F_{p}^{(\alpha, \beta)}($.$) . On account of the most general nature of the F_{p}^{(\alpha, \beta)}($.$) a large number$ of sequences and polynomials involving simpler functions can be easily obtained as their special cases but due to lack of space we can not mention here.

\section{REFERENCES}

[1] Agarwal, P., Chand, M., (2013), On new sequence of functions involving ${ }_{p} F_{q}$, South Asian Journal of Mathematics, Vol. 3 ( 3 ) : 199-210. 
[2] Agarwal, P., Chand, M., (2013), A new sequence of functions involving $p_{j} F_{q_{j}}$, Mathematical Sciences And Applications E-Notes, Volume 1 No. 2 pp. 173-190.

[3] Agarwal, P., Chand, M.,(2013), Graphical Interpretation of the New Sequence of Functions Involving Mittage-Leffler Function Using Matlab, American Journal of Mathematics and Statistics 2013, 3(2): 73-83 DOI: 10.5923/j.ajms.20130302.02.

[4] Agarwal, P., Chand, M. and Dwivedi, S.,(2014), A Study on New Sequence of Functions Involving $\overline{\mathrm{H}}$-Function, American Journal of Applied Mathematics and Statistics, Vol. 2, No. 1, 34-39.

[5] Chak, A. M., (1956) A class of polynomials and generalization of stirling numbers, Duke J. Math., 23, 45-55.

[6] Chandel, R.C.S., (1973) A new class of polynomials, Indian J. Math., 15(1), 41-49.

[7] Chandel, R.C.S., (1974) A further note on the class of polynomials $T_{n}^{\alpha, k}(x, r, p)$, Indian J. Math.,16(1), 39-48.

[8] Chatterjea, S. K., (1964) On generalization of Laguerre polynomials, Rend. Mat. Univ. Padova, 34, 180-190.

[9] Gould, H. W. and Hopper, A. T., (1962) Operational formulas connected with two generalizations of Hermite polynomials, Duck Math. J., 29, 51-63.

[10] Joshi, C. M. and Prajapat, M. L., (1975) The operator $T_{a, k}$, and a generalization of certain classical polynomials, Kyungpook Math. J., 15, 191-199.

[11] Mittal, H. B., (1971) A generalization of Laguerre polynomial, Publ. Math. Debrecen, 18, 53-58.

[12] Mittal, H. B., (1971) Operational representations for the generalized Laguerre polynomial, Glasnik Mat.Ser III, 26(6), 45-53.

[13] Mittal, H. B., (1977) Bilinear and Bilateral generating relations, American J. Math., 99, $23-45$.

[14] Özergin, E., Some properties of hypergeometric functions,Ph.D. Thesis, Eastern Mediterranean University, North Cyprus, February 2011.

[15] Patil, K. R. and Thakare, N. K., (1975) Operational formulas for a function defined by a generalized Rodrigues formula-II, Sci. J. Shivaji Univ. 15, 1-10.

[16] Shrivastava, P. N., (1974) Some operational formulas and generalized generating function, The Math. Education, 8, 19-22.

[17] Shukla, A. K. and Prajapati J. C., (2007) On some properties of a class of Polynomials suggested by Mittal, Proyecciones J. Math., 26(2), 145-156.

[18] Srivastava, H. M. and Choi,J., (2012) Zeta and q-Zeta Functions and Associated Series and Integrals, Elsevier Science Publishers, Amsterdam, London and New York.

[19] Srivastava, A. N. and Singh, S. N., (1979) Some generating relations connected with a function defined by a Generalized Rodrigues formula, Indian J. Pure Appl. Math., 10(10), 1312-1317.

[20] Srivastava, H. M. and Singh, J. P., (1971) A class of polynomials defined by generalized, Rodrigues formula, Ann. Mat. Pura Appl., 90(4), 75-85.

[21] Wright, E.M., (1935a) The asymptotic expansion of the generalized hypergeometric function. J. London Math. Soc. 10. 286-293.

[22] E. Özergin, Some properties of hypergeometric functions,Ph.D. Thesis, Eastern Mediterranean University, North Cyprus, February 2011.

[23] E. Özergin, M. A. Özarslan and A. Altin, Extension of gamma, beta and hypergeometric functions, J. Comput. Appl. Math. 235(2011), 4601-4610.

(P. Agarwal) Department of Math., Anand International College of Engineering, JAIPUR-303012, INDIA

E-mail address: goyal.praveen2011@gmail.com

(S. Jain) Department of Math., Poornima College of Engineering, Sitapura Jaipur, INDIA

E-mail address: shilpijain1310@gmail.com

(İ. O. Kiymaz) Department of Math., Ahi Evran University, 40100, Kirşehir, Turkey

E-mail address: ioiymaz@ahievran.edu.tr

(M. Chand) Department of Math., Fateh College for Women, Bathinda-151001, India E-mail address: mehar.jallandhra@gmail.com 
(S.K.Q. Al-Omari) Department of Applied Sciences, Faculty of Engineering Technology, Al-BalqA' Applied University, Amman 11134, Jordan

E-mail address: s.k.q.alomari@fet.edu.jo 